\title{
Cell cycle arrest biomarkers for the early prediction of acute kidney injury - full of promise, but not a must-have for yet
}

\author{
Helmut Schiffl ${ }^{*}$ \\ Department of Internal Medicine IV, University Hospital Munich, Munich, Germany
}

\section{A R T I C L E I N F O}

\section{Article Type:}

Review

\section{Article History:}

Received: 4 October 2016

Accepted: 24 November 2016

Published online: 6 December 2016

\section{Keywords:}

Acute kidney injury

Outcome

Cell cycle arrest biomarker

\begin{abstract}
A B S T R A C T
Hospital acquired acute kidney injury (AKI) is one of the most common organ dysfunctions in elderly and critically ill patients and adversely impacts on short-term as well as on longterm clinical outcomes. Early diagnosis of AKI, preferably within 12 to 24 hours after onset of the renal insult, is pivotal to the development of effective therapies attenuating acute kidney dysfunction severity or fastening renal recovery. Traditional biomarkers like creatinine are late indicators of AKI. The cell cycle arrest biomarkers (CCABs) tissue inhibitor of metalloproteinase-2 and insulin like growth factor binding protein-7 accurately predicted moderate to severe AKI in high-risk cardiothoracic surgery patients within 12 hours. These CCABs outperformed the most promising current biomarkers. The NephroCheck test system represents a promising tool inching closer to widespread clinical application and received FDA approval in September 2014. However, there is an urgent need to demonstrate the costeffectiveness of this biomarker test system whether alone or in combination with biomarkers of other pathways. Most importantly, further trials should validate the cut-off values in patient populations other than intensive care unit (ICU) and cardiac surgery.
\end{abstract}

Implication for health policy/practice/research/medical education:

The cell cycle arrest biomarkers (CCABs) tissue inhibitor of metalloproteinase- 2 and insulin like growth factor binding protein -7 accurately predicted moderate to severe acute kidney injury (AKI) in high-risk cardiothoracic surgery patients within 12 hours. These CCABs outperformed the most promising current biomarkers. The NephroCheck test system represents a promising tool inching closer to widespread clinical application and received FDA approval in September 2014. However, there is an urgent need to demonstrate the cost-effectiveness of this biomarker test system whether alone or in combination with biomarkers of other pathways. Most importantly, further trials should validate the cut-off values in patient populations other than intensive care unit (ICU) and cardiac surgery.

Please cite this paper as: Schiffl H. Cell cycle arrest biomarkers for the early prediction of acute kidney injury - full of promise, but not a must-have for yet. J Renal Inj Prev. 2017;6(3):177-183. DOI: 10.15171/jrip.2017.34.

\section{Introduction}

Conceptually, acute kidney injury (AKI) is defined as an abrupt (over hours to days) decrease in glomerular filtration rate (GFR), resulting in retention of creatinine, urea and other waste products and in dysregulation of body fluid, electrolyte and acid-base homeostasis. Clinically, this disorder has multiple etiologies and risk factors, encompasses a broad spectrum of manifestations ranging from tubular stress to oligo-anuric renal failure and is associated with unpredictable outcomes. AKI is a common problem amongst hospitalized patients, especially in elderly patients whose numbers are increasing and in those treated in the intensive care unit (1). This renal disorder is no longer considered an innocent bystander merely reflecting the severity of precipitating acute illness or of coexisting pathologies. Irrespective of its nature, severity and duration AKI is an independent and strong determinant of in hospital mortality, intensive care unit (ICU) readmission and post-discharge renal and cardiovascular morbidity and mortality. At present, prevention of AKI focuses on the optimization of cardiac filling pressure to improve renal perfusion and on the avoidance of exposure to nephrotoxins. Management of established AKI is primarily supportive. In spite of 
many advances in intensive care and renal replacement techniques, the 10-year survival rate of critically ill patients with AKI severe enough requiring RRT is only $20 \%(2-4)$.

\section{Materials and Methods}

For this review, we applied a diversity of sources by searching through PubMed/Medline, Scopus, EBSCO, Embase, Web of Science, and directory of open access journals (DOAJ). The search was conducted using the combination of the following key words and or their equivalents such as acute kidney injury, outcome and Cell cycle arrest biomarker.

\section{Conventional biomarkers of AKI}

Acute kidney injury is considered a silent disease that commonly occurs in patients with acute illness. Its early stages cause few specific symptoms or clinical signs. Currently, simple surrogate markers of GFR and urinalysis identify AKI. However, the standard metrics used to define AKI and to monitor its progression, such as serum creatinine or blood urea nitrogen levels, are insensitive, nonspecific and are increased only after significant kidney injury (more than half loss of the kidney function) and then with a substantial time delay. Increases in serum creatinine levels represent a delayed (hours to days) indication of a functional change in GFR that lags behind structural changes that occur during the early stages of AKI. The same increment in serum creatinine may have different clinical implications. Single point measurements demonstrating increased serum creatinine do not allow differentiation between pre-renal azotemia, intrinsic renal dysfunction and obstructive uropathy, nor do they differentiate between AKI and chronic kidney disease. Finally, subclinical renal injury occurs without overt changes in serum creatinine (5).

Urine output, another diagnostic marker of AKI, is unreliable except in monitored settings such as intensive care units and can be altered by administration of fluid and diuretics. Urine chemistry such as fractional excretion rate of sodium or urea may differentiate pre-renal azotemia from acute tubular necrosis in selected patients. However, administration of diuretics, changes in volume status and different causes of AKI may confound the results of these tests. Furthermore, microscopy of the urine sediment for renal tubular casts may be helpful in differentiating prerenal azotemia from acute tubular necrosis. However, many centers lack experienced personnel to interpret urine microscopy.

The delayed or even missed diagnosis of AKI by the use of conventional biomarkers prevents timely patient management decisions, including the administration of putative renoprotective agents. The National Confidential Enquiry into Patient Outcomes and Death Report demonstrated that $30 \%$ of the patients had predictable and avoidable AKI. Early recognition and successful preventive measures would result in a large reduction of deaths, complications and costs due to AKI (6).

\section{Novel biomarkers of AKI}

In most forms of acute kidney, structural changes precede functional impairment. Ideally, kidney specific biomarkers of AKI should identify patients at risk and evaluate the susceptibility of the kidneys to toxic exposures. They should allow an early recognition of renal injury/dysfunction (i.e. before alterations in traditional markers occur), enable differential diagnosis, stratify patients based on prognosis (progression, need for renal replacement therapy, recovery, survival) and to monitor the response to interventions and treatment. The integration of novel biomarkers into clinical practice should enable incremental diagnostic and prognostic information beyond that found by clinical evaluation or traditional markers alone $(5,7)$.

Numerous different urinary and serum proteins, molecules and - most recently - micro RNAs have been rigorously investigated during the last decade.

The rise and fall of promising biomarkers of AKI While novel AKI biomarkers have enhanced our understanding of the pathophysiologic processes during AKI, the clinical utility of most of them is not clear. Countless investigations have identified biomarkers that facilitate the early diagnosis, differential diagnosis and short- and long-term prognosis of pediatric patients after timed injury (8). Such markers are urinary neutrophil gelatinase-associated lipocalin (NGAL), urinary interleukin 18 (IL-18), plasma and urinary cystatin C, urinary kidney injury molecule 1 (KIM-1) or urinary liver type fatty acid binding protein (L-FABP). Unfortunately, the performance of these novel biomarkers in adult patients with sepsis or comorbidities is not robust. Results are influenced by patient case mix, comorbidities, type of kidney insult, timing of measurements as well as selected thresholds for diagnosis and prognosis of AKI. Particularly, intensive care patients at risk for AKI suffer from a strong inflammatory response with injury of several organ systems. They are exposed to repeated diagnostic and therapeutic procedures, fluid therapy, vasopressor support, and multiple other pharmacologic substances. There is no surprise that specific biomarkers show a major loss of diagnostic accuracy or predictive ability when used in critically ill patients, where "biomarkeremia" and/ or "biomarkeruria" may be a biological signal of overall illness severity rather than specific kidney damage $(5,7)$. Recently, monitoring of urinary concentrations of tissue inhibitor of metalloproteinase-2 (TIMP-2) and insulin -like growth factor binding protein-7 (IGFBP 7) might serve as an early method to detect AKI following cardiac surgery. The combination of these two biomarkers seems to be highly predictive of which patients will develop moderate to severe AKI in the next 12-24 hours. These two G1 cell-cycle arrest biomarkers (CCABs) outperformed the other most studied markers of AKI. The test system for CCABs (NephroCheck, Astute Medical Inc., San Diego, USA) received US Food and Drug Administration 
(FDA) approval in September 2014. The FDA-approved indication for the test specifies "to be used in conjunction with clinical evaluation in patients who currently have, or have had within the past 24 hours, acute cardiovascular and/or respiratory compromise" and are ICU patients, as an aid in the risk assessment for moderate or severe AKI within 12 hours of patient assessment. The test is intended for use in adult patients, in conjunction with other clinical and laboratory tests, and is not intended as a standard test (9).

Discovery and validation of cell cycle arrest biomarkers of AKI

The primary evidence on the CCABs comes from four large multicenter studies conducted in Europe and North America. These studies are named Discovery (10), Sapphire (10), Opal (11) and Topaz (12).

The Discovery trial was conducted at three clinical sites and included 522 patients (median age 64 years, 91\% Caucasian, sepsis, shock, major surgery and trauma). Three hundred forty biomarkers were screened and ranked both individually and in combination according to their ability to predict moderate or severe AKI (RIFLE stages I or F) within 12 to 36 hours (10). TIMP2 and IGFBP-7 were the best-performing markers. The area under the receiver operating characteristic curve (AUC) were 0.77 and 0.75 , respectively (with $\mathrm{AUC}=1.0$ maximum performance and $\mathrm{AUC}=0.5$ chance performance) and were therefore validated in the Sapphire study (10).

The Sapphire study was performed in 35 centers and included 728 critically ill patients (median age 64 years, chronic kidney disease and sepsis each in 19\%). Moderate to severe AKI occurred in $14 \%$ of the patients. The inducers of G1 cell cycle arrest together demonstrated an AUC of 0.8 (0.76 and 0.79 alone). None of preexisting markers achieved an AUC better than 0.72. The CCABs improved risk stratification when added to a nine variable clinical model (10). The results of the Sapphire study were used to establish a cut-off threshold for detecting AKI risk as $0.3(\mathrm{ng} / \mathrm{mL})^{2} / 1000$ which was prospectively conformed in the Opal study (11).

The Opal study was intended to validate the biomarker cut-off values 0.3 and $2.0(\mathrm{ng} / \mathrm{mL})^{2} / 1000$ for sensitivity, specificity and relative risk to predict development of moderate to severe AKI (KDIGO stages 2 or 3 ) within 12 hours of testing. The study compared data from the Sapphire study with data from a cohort of 154 critically ill adult US patients (9\% had a history of chronic kidney disease, sepsis was the main cause for ICU admission in 19\%). The results were comparable for both study populations. The AUC was 0.8 for Sapphire and 0.79 for Opal. Estimates of sensitivity were $89 \%$ at the 0.3 cut-off in both patient groups, while specificity estimates were 50 $\%$. At the 2.0 cut-off estimates of sensitivity were $42 \%$ and $44 \%$ respectively. Specificity estimates were $95 \%$ and $90 \%$ : The very high NPV (negative predictive value) of $97 \%$ for the low 0.3 cut-off predicted that almost all patients tested negative for the test would be free of AKI for the next 12 hours.

The prospective multicenter Topaz trial included 420 critically ill patients with severe respiratory or cardiovascular disease (9\% had chronic kidney disease, sepsis was the main cause of ICU admission in 24\%). The trial assessed the ability of NephroCheck biomarkers to predict patients at risk of developing moderate to severe AKI within 12 hours of testing (12). At the 0.3 cut-off value the CCABs test sensitivity was $92 \%$. When the biomarker results were combined with clinical variables, the AUC was 0.86 vs. 0.7 for clinical parameters alone, and 0.82 for the biomarker test alone. At the 0.3 cut-off values, specificity for the biomarker test was $0.46 \%$.

A follow up study of a subset of 692 patients from the Sapphire trial found that patients with AKI and early CCABs scores of 2.0 had an increased risk of death or of requiring RRT during the following nine months compared with a score of 0.3 (13).

Secondary analyses of 375 adult surgical ICU participants of the Sapphire trial and the Topaz trial found that a single urinary TIMP-2×IGFBP7 test accurately identified patients at risk for developing AKI. Its inclusion in clinical risk models significantly enhanced their performance (14).

Beyond the four primary studies described above, smaller studies of these biomarkers in adult or pediatric AKI have been published (15-19) with more or less promising data.

Reference intervals of urinary cell cycle arrest AKI markers in healthy subjects and chronic comorbid subjects without AKI

A recent multi-site trial determined the reference intervals of these biomarkers in apparently healthy adults $(\mathrm{N}=378)$ and subjects with at least one stable chronic morbidity $(\mathrm{N}=372$, renal insufficiency in $1.6 \%$ of the patients). The reference values in apparently healthy subjects and subjects with stable chronic morbidity were 0.04-2.25 and $0.05-2.20(\mathrm{ng} / \mathrm{mL})^{2} / 1000$ respectively. There was no statistical difference in the reference interval among the two study populations. However, reference values in healthy subjects and stable chronic patients overlapped with the values obtained from AKI patients. Reference interval data showed that overall $50 \%$ of the study population had values above 0.33 . The authors stated that CCABs values obtained from critically ill patients should be used in conjunction with the patient condition and clinical signs or symptoms and that the test was developed to assess risk of AKI and not intended as a sole indicator for the diagnosis of AKI $(20,21)$.

Conflicting data on the precision of NephroCheck A German study included 42 patients undergoing cardiac bypass surgery and analyzed whether urinary TIMP-2 and IGFBP7 were adequate diagnostic tests to identify early AKI after cardiac surgery (22). Thirty-eight percent of the study patients experienced AKI. The results showed 
a median baseline concentration of $0.3(\mathrm{ng} / \mathrm{mL})^{2} / 1000$, which decreased at the end of surgery and then increased at 4 hours after surgery. Further increases were noted on the first post-operative day with significantly higher CCABs concentrations in patients with AKI. However, on the day of surgery, the concentrations did not differ between patients classified as KDIGO 0 or KDIGO 1 or 2. The authors found, that their study did not confirm previously published cut-off points of 0.3 and 2 (ng/ $\mathrm{mL})^{2} / 1000$. The sensitivity and specificity of the cut-off point of 0.3 were $53 \%$ and $54 \%$ respectively. The cutoff point of $2(\mathrm{ng} / \mathrm{mL})^{2} / 1000$ led to a sensitivity of $33 \%$ and a specificity of $100 \%$.

Numerous factors may explain these discrepant results; 1 ) The German study was not restricted to patients at high risk for AKI. There are a number of examples of biomarker performance degradation when cohorts include highrisk patients as well as affected patients. 2) The surgical fluid management practice differed from the validation trials and resulted in a post-operative decline (dilution) of the biomarker concentrations. 3) The study had a small sample size and few patients with post-cardiac surgery AKI. With only 16 of 43 enrolled patients reaching the endpoint of AKI this study has a power well below $60 \%$ rendering any conclusion regarding test performance statistically meaningless.

A second single-center German study of 50 patients undergoing cardiac bypass (CBP) surgery measured urinary CCABs at 4, 12, 24, 48 and 72 hours after CBP (18). These investigators found a marked rise of these biomarker values at all points in time in the 26 patients. Twenty-six patients (52\%) developed post-cardiac surgery AKI. A composite of the maximum urinary CCABs concentrations during the first 24 hours was superior to measurements at each individual time point, with an AUC of 0.9. At four hours, the biomarker test AUC was 0.81 . By comparison, another urinary biomarker test, NGAL at four hours had an AUC of 0.68.

A recent Swedish study including 94 ICU patients with normal renal function assessed CCABs to predict early and delayed AKI (23). Urine samples were collected within 48 hours of ICU admission and no AKI at the beginning of sampling. Nineteen patients (20\%) developed AKI within 48 hours (8 patients within 12 hours, 4 patients within 1224 hours, and 7 patients within 24-48 hours). The urinary biomarkers levels (CCABs, NGAL and cystatin) in non AKI patients and in patients who developed AKI within 12-to 48 hours did not differ significantly. Diabetes was independently associated with higher CCABs, but AKI or sepsis were not. The authors concluded that urinary CCABs (as well as NGAL or cystatin) should be used cautiously as AKI predictors in general ICU patients since urine levels of these biomarkers are affected by factors other than AKI and their performance can be poor. The strengths of this study are that it applied biomarker testing to a general ICU patient population with detailed information on septic state, level of AKI as well as demographic and comorbidity data. Noteworthy, the authors used a point-of care commercial kit for CCABs measurements.

\section{Limitations of published investigations}

Admittedly, the discovery and validation trials of cell arrest biomarkers have shown an outstanding performance of the two CCABs IGFBP 7 and TIMP-2 in selected ICU patients (acute hemodynamic or respiratory compromise) with high risk for post-cardiac surgery AKI. However, there has not been published much about the day to day clinical use of the NephroCheck test in other hospitalized patients. Whether or not the test performs equally in unselected cohorts, like mixed ICU patient populations, or patients treated in the emergency room or in general hospital wards remains to be demonstrated.

Regrettably, the few efforts of small studies to reproduce the results of the validation trials have been controversial. There are notable differences in the performance of these biomarkers between Sapphire (10) and Opal (11) trials on the one hand and the studies reported by Bell et al (23) and Wetz et al (22) on the other hand, even though both were done in ICU patients. These conflicting findings suggest that the performance of the cell cycle arrest markers may decrease markedly in unselected critically ill patients. There are numerous examples of performance degradation of novel biomarkers (initial AUC above 0.9) resulting in questionable utility with AUCs frequently below 0.7 .

Generally, critical problems affecting biomarkers in heterogeneous patient populations involve the cause of AKI, context (co-morbid diseases and baseline renal function), timing of the injury and timing and/or number of samples. The two CCABs have been evaluated in patients with cardiothoracic surgery, with a predominance of Caucasians, male gender as well as nephrotoxic or ischemic AKI. The reported biomarkers performed well in patients with sepsis (AUC 0.82) and post-cardiac surgery (AUC 0.85). IGFBP 7 may be superior to TIMP-2 in surgical patients while TIMP 2 is best in sepsis-induced AKI. However, differences in the number of patients alone may not explain divergent results among investigations with cardiac surgery patients, but also differences in patient cohort composition and surgical procedures. Compared to cardiac surgical procedures with valve or combined valve replacement, coronary artery bypass grafting only surgery requires shorter cardiopulmonary bypass and aortic clamp time, assuming less ischemic injury to the kidneys. The risk of AKI could have been considerably higher in the study by Meersch et al (18) than in the study by Wetz et al (22), because they also included patients undergoing valvular and combined cardiac surgery with significantly longer cardiopulmonary bypass and aortic clamp times. The low-grade AKI cohort of Wetz et al may be a major reason why these authors could not confirm previously cutoff points.

Currently, cut-off values established for NephroCheck in the validation trials and specified in the US FDA approved 
indication have not been validated in populations such as newborns, children, patients with pre-existing kidney disease or patients receiving contrast media or nephrotoxic antibiotics. There is circumstantial proof, that the optimal test threshold for novel biomarkers may vary for different co-morbidities and settings. The same statement may hold true for the new CCABs.

The US FDA approval noted the relatively low specificity (around 50\%) of the low cutoff values. A significant proportion of patients who are not at risk of AKI will have a positive test result and emphasized that the test should be used in conjunction with other clinical criteria to assess AKI risk. The number of positive biomarker tests without consecutive rise in serum creatinine may be magnified in populations at low risk for AKI (24). The demonstration that novel biomarkers may be elevated without overt changes in serum creatinine changes is not limited to CCABs. A meta-analysis of aggregate data from 2322 patients (10 studies) showed that $19.2 \%$ of patients had raised NGAL levels without relevant changes in serum creatinine. Mortality, length of stay in the ICU and need for RRT were significantly higher in the cohort with increased NGAL levels than in patients without an urinary NGAL and serum creatinine rise (25). Whether or not novel biomarkers in these circumstances are an indicator for subclinical renal injury or simply a marker of illness severity remains unclear. However, the high number of positive tests in unselected patients can potentially lead to potential harm if important interventions (antibiotics, contrast media) are given delayed or withheld on the base of false positive biomarker results.

The timing and number of urine collections in the validation trials warrant a comment. In contrast to cardiac surgery or administration of contrast media or antibiotics, it is often difficult to determine the actual onset of AKI. Additionally the kinetics of CCABs are unknown in some patient populations. Significant biomarker dilution is likely in the immediate post-cardiac surgery setting and in patients with cardiac arrest. The primary analysis of the Sapphire (10) and Topaz (12) trials was the ability of urinary TIMP- $2 \times$ IGFBP 7 to predict moderate to severe AKI within 12 hours after ICU admission. While most of the subjects in the Opal trial were recruited prior to clinical manifestations of AKI, some patients were enrolled with elevated or maximal serum creatinine concentrations on admission. One-hundred and one patients of the Sapphire cohort met the primary end-point (moderate to severe AKI) within 12 hours. However, in addition 218 individuals (30\%) developed AKI within 7 days (10). The diagnostic window of 12 hours leads to an early prediction of AKI in high risk patients, but single point measurements of these biomarkers will miss twothirds of patients with post-cardiac surgery.

The NephroCheck test is intended for use in adult patients in conjunction with other clinical or laboratory tests. This test does not replace the clinical experience of nephrologists. However, it is largely unknown whether these CCABs add anything beyond clinical evaluation combined with traditional renal function tests. In a multivariate model including clinical information, urinary TIMP-2 x IGFBP-7 remained statistically significant and a strong predictor of AKI (AUC 0.7 for clinical variables alone vs. clinical variables plus TIMP-2 and IGFBP7, AUC 0.86). The clinical model used in the validation trial included preexisting chronic kidney disease, APACHE III score, sepsis, nephrotoxic dugs, hypotension, liver disease and diabetes. Amazingly, they did not include cardiopulmonary bypass or aortic clamp time, brain natriuretic peptide (26), post-operative diuresis or furosemide stress test (13), New York Heart Association (NYHA) classification of heart failure or other recognized risk factors.

Up to now, novel biomarkers including CCABs have been validated against serum creatinine, since absolute or relative increases in creatinine are the primary basis for the consensus definitions of AKI, although the changes actually are surrogates for changes in the GFR. Thus for validity, damage markers need to be tested against an independent gold standard. However, renal histology is not routinely available. Whether the sensitivity for RT or death can be used to identify biomarker cutoffs for AKI diagnosis remains unclear.

\section{Crucial questions remain unanswered}

Early detection of AKI could trigger more aggressive volume management and differences in the dosing of medications such as antibiotics. Currently, there is no directed therapy for AKI. Many previously tested therapeutics may have failed because they simply did not work, some may have failed because the inability to get the drug into the patient within the right window of time. Therapeutics may be more easily tested and their values more accurately assessed by identifying high-risk patients closer to the time of potential kidney injury. No biomarker had yet shown the efficacy of any intervention based on increased biomarkers alone. The only intervention study using novel biomarkers to guide treatment was negative. Endre and colleagues performed a double blind placebo controlled study to investigate whether early treatment with high erythropoietin doses could prevent the development of clinically diagnosed AKI in ICU patients (27). The decision to randomize patients was based solely on the use of a threefold increase in urinary y-GT. Unfortunately, early intervention with erythropoietin was not successful in preventing AKI, renal replacement therapy or death. Secondly, a German multicenter randomized trial investigated the effect of remote ischemic preconditioning (RIP) on post-cardiac surgery AKI. Two-hundred forty patients at high risk for AKI were randomized in a 1:1 ratio to receive RIP or sham RIP. RIP had no significant effect on myocardial infarction, stroke or mortality, but was associated with a significant reduction in AKI, less RRT and shorter ICU stay, as well as a significant attenuation in the release of IGFBP 7 and TMP-2 after surgery (28). 
In contrast, a meta-analysis including 2200 study patients, found no significant difference in postoperative kidney function (serum creatinine or GFR,), incidence of RRT, or hospital mortality, and hospital or ICU stay in patients receiving RIP (29). Thus, adequately powered trials are needed to prove the evidence of a beneficial effect of RIP for prevention of post-cardiac surgery AKI. Finally, it may be attractive to know which patient will need RRT, but earlier knowledge is unlikely to change clinical practice. There is no evidence that initiation of RRT earlier than by current criteria is beneficial (30).

\section{Conclusion}

The increasing number of AKI patients, their rising age, and number of co-morbidities and also severity of acute illness, all make earlier detection of AKI and timely intervention for patient care necessary. The NephroCheck biomarkers performed better than other novel biomarkers in selected ICU patients at high risk for post cardiac surgery AKI for a 12-hour time frame. Nevertheless, the predictive ability of the CCABs is just beginning to be elucidated. Further studies are needed to detect the value of this test alone or combined with other biomarkers or clinical parameters across different patient populations and to define its place within patient care.

\section{Author's contribution}

HS is the single author of the manuscript.

\section{Conflicts of interest}

The author declare no conflict of interest.

\section{Ethical considerations}

Ethical issues (including plagiarism, data fabrication, double publication) have been completely observed by the author.

\section{Funding/Support}

No funding was received for this study.

\section{References}

1. Hoste EA, Bagshaw SM, Bellomo R, Cely CM, Colman $\mathrm{R}$, Cruz DN, et al. Epidemiology of acute kidney injury in critically ill patients: the multinational AKI-EPI study. Intensive Care Med. 2015;41:1411-23. doi: 10.1007/s00134015-3934-7.

2. Bellomo R, Kellum JA, Ronco C. Acute kidney injury. Lancet. 2012;380:756-66.

3. Chawla LS, Kimmel PL. Acute kidney injury and chronic kidney disease: an integrated clinical syndrome. Kidney Int. 2012;82:516-24. doi: 10.1038/ki.2012.208.

4. Schiffl H, Lang SM, Fischer R. Long-term outcome of survivors of ICU acute kidney injury requiring renal replacement therapy: a ten year prospective cohort study. Clin Kidney J. 2012;5:297-302.

5. Schiffl H, Lang SM. Update on biomarkers of acute kidney injury: moving closer to clinical impact? Mol Diagn Ther. 2012;16:199-207. doi: 10.2165/11634310-00000000000000 .
6. MacLeod A. NCEPOD report on acute kidney injury-must do better. Lancet. 2009;374:1405-6. doi: 10.1016/S01406736(09)61843-2.

7. Ostermann M, Joannidis M. Biomarkers for AKI improve clinical practice: no. Intensive Care Med. 2015;41:618-22. doi: 10.1007/s00134-014-3540-0.

8. Schiffl H, Lang SM. Urinary biomarkers and acute kidney injury in children: the long road to clinical application. Pediatr Nephrol. 2013;28:837-42.

9. Endre ZH, Pickering JW. Acute kidney injury: cell cycle arrest biomarkers win race for AKI diagnosis. Nat Rev Nephrol. 2014;10(12):683-5. doi: 10.1038/nrneph.2014.198.

10. Kashani K, Al-Khafaji A, Ardiles T, Artigas A, Bagshaw SM, Bell M, et al. Discovery and validation of cell cycle arrest biomarkers in human acute kidney injury. Crit Care. 2013;17:R25. doi: 10.1186/cc12503.

11. Hoste EA, McCullough PA, Kashani K, Chawla LS, Joannidis $\mathrm{M}$, Shaw AD, et al. Derivation and validation of cutoffs for clinical use of cell cycle arrest biomarkers. Nephrol Dial Transplant. 2014;29:2054-61. doi: 10.1093/ndt/gfu292.

12. Bihorac A, Chawla LS, Shaw AD, Al-Khafaji A, Davison DL, Demuth GE, et al. Validation of cell-cycle arrest biomarkers for acute kidney injury using clinical adjudication. Am J Respir Crit Care Med. 2014;189:932-9. doi: 10.1164/ rccm.201401-0077OC.

13. Koyner JL, Shaw AD, Chawla LS, Hoste EA, Bihorac A, Kashani $\mathrm{K}$, et al. Tissue Inhibitor Metalloproteinase-2 (TIMP-2)IGF-Binding Protein-7 (IGFBP7) Levels Are Associated with Adverse Long-Term Outcomes in Patients with AKI. J Am Soc Nephrol. 2015;26:1747-54. doi: 10.1681/asn.2014060556.

14. Gocze I, Koch M, Renner P, Zeman F, Graf BM, Dahlke $\mathrm{MH}$, et al. Urinary biomarkers TIMP-2 and IGFBP7 early predict acute kidney injury after major surgery. PLoS One. 2015;10:e0120863. doi: 10.1371/journal.pone.0120863.

15. Westhoff JH, Tonshoff B, Waldherr S, Poschl J, Teufel $\mathrm{U}$, Westhoff $\mathrm{TH}$, et al. Urinary tissue inhibitor of metalloproteinase-2 (TIMP-2)*insulin-like growth factorbinding protein 7 (IGFBP7) predicts adverse outcome in pediatric acute kidney injury. PLoS One. 2015;10:e0143628. doi: 10.1371/journal.pone.0143628

16. Pajenda S, Ilhan-Mutlu A, Preusser M, Roka S, Druml W, Wagner L. NephroCheck data compared to serum creatinine in various clinical settings. BMC Nephrol. 2015;160:206. doi: 10.1186/s12882-015-0203-5.

17. Pilarczyk K, Edayadiyil-Dudasova $M$, Wendt D, Demircioglu E, Benedik J, Dohle DS, et al. Urinary [TIMP2]*[IGFBP7] for early prediction of acute kidney injury after coronary artery bypass surgery. Ann Intensive Care. 2015;5:50. doi: 10.1186/s13613-015-0076-6.

18. Meersch M, Schmidt C, Van Aken H, Martens S, Rossaint J, Singbartl K, et al. Urinary TIMP-2 and IGFBP7 as early biomarkers of acute kidney injury and renal recovery following cardiac surgery. PLoS One. 2014;9:e93460. doi: 10.1371/journal.pone.0093460.

19. Gunnerson KJ, Shaw AD, Chawla LS, Bihorac A, Al-Khafaji A, Kashani K, et al. TIMP2*IGFBP7 biomarker panel accurately predicts acute kidney injury in high-risk surgical patients. J Trauma Acute Care Surg. 2016;80:243-9. doi: 10.1097/ta.0000000000000912.

20. Chindarkar NS, Chawla LS, Straseski JA, Jortani SA, Uettwiller-Geiger D, Orr RR, et al. Demographic data for urinary acute kidney injury (AKI) marker [IGFBP7]. 
[TIMP2] reference range determinations. Data Brief. 2015;5:888-92. doi: 10.1016/j.dib.2015.10.036.

21. Chindarkar NS, Chawla LS, Straseski JA, Jortani SA, Uettwiller-Geiger D, Orr RR, et al. Reference intervals of urinary acute kidney injury (AKI) markers [IGFBP7] [TIMP2] in apparently healthy subjects and chronic comorbid subjects without AKI. Clin Chim Acta. 2016;452:32-7. doi: 10.1016/j.cca.2015.10.029.

22. Wetz AJ, Richardt EM, Wand S, Kunze N, Schotola H, Quintel M, et al. Quantification of urinary TIMP-2 and IGFBP-7: an adequate diagnostic test to predict acute kidney injury after cardiac surgery? Crit Care. 2015;19:3. doi: 10.1186/s13054-014-0717-4.

23. Bell M, Larsson A, Venge P, Bellomo R, Martensson J. Assessment of cell-cycle arrest biomarkers to predict early and delayed acute kidney injury. Dis Markers. 2015;2015:158658. doi: 10.1155/2015/158658.

24. Vijayan A, Faubel S, Askenazi DJ, Cerda J, Fissell WH, Heung M, et al. Clinical use of the urine biomarker [TIMP2] $x$ [IGFBP7] for acute kidney injury risk assessment. Am J Kidney Dis. 2016;68:19-28. doi: 10.1053/j.ajkd.2015.12.033.

25. Haase M, Devarajan P, Haase-Fielitz A, Bellomo R, Cruz DN, Wagener G, et al. The outcome of neutrophil gelatinase-associated lipocalin-positive subclinical acute kidney injury: a multicenter pooled analysis of prospective studies. J Am Coll Cardiol. 2011;57:1752-61. doi: 10.1016/j. jacc.2010.11.051.

26. Chou YH, Chen YF, Pan SY, Huang TM, Yang FJ, Shen WC, et al. The role of brain natriuretic peptide in predicting renal outcome and fluid management in critically ill patients. J Formos Med Assoc. 2015;114:1187-96. doi: 10.1016/j. jfma.2015.10.015.

27. Endre ZH, Walker RJ, Pickering JW, Shaw GM, Frampton $\mathrm{CM}$, Henderson SJ, et al. Early intervention with erythropoietin does not affect the outcome of acute kidney injury (the EARLYARF trial). Kidney Int. 2010;77:1020-30. doi: 10.1038/ki.2010.25.

28. Zarbock A, Schmidt C, Van Aken H, Wempe C, Martens S, Zahn PK, et al. Effect of remote ischemic preconditioning on kidney injury among high-risk patients undergoing cardiac surgery: a randomized clinical trial. JAMA. 2015;313:2133-41. doi: 10.1001/jama.2015.4189.

29. Healy DA, Khan WA, Wong CS, Moloney MC, Grace PA, Coffey JC, et al. Remote preconditioning and major clinical complications following adult cardiovascular surgery: systematic review and meta-analysis. Int J Cardiol. 2014;176:20-31. doi: 10.1016/j.ijcard.2014.06.018.

30. Davenport A. Early start renal replacement therapy for acute kidney injury-Universal panacea or another case of over medicalization? Hemodial Int. 2015;19 Suppl 3:S34-9. doi: $10.1111 /$ hdi.12351.

Copyright $\odot 2017$ The Author(s); Published by Nickan Research Institute. This is an open-access article distributed under the terms of the Creative Commons Attribution License (http://creativecommons.org/licenses/by/4.0), which permits unrestricted use, distribution, and reproduction in any medium, provided the original work is properly cited. 